Proceedings

\title{
Optimization of Ultrasound Assisted Extraction of Phenolic Compounds from Orange By-Product ${ }^{+}$
}

\author{
María del Carmen Razola-Díaz 1,*, Eduardo Jesús Guerra-Hernández ${ }^{1}$, Celia Rodríguez-Pérez 2,3, \\ Ana María Gómez-Caravaca ${ }^{4}$, Belén García-Villanova ${ }^{1}$ and Vito Verardo ${ }^{1,4}$ \\ 1 Department of Nutrition and Food Science, University of Granada, Campus of Cartuja, 18011 Granada, \\ Spain; ejguerra@ugr.es (E.J.G.-H.); belenv@ugr.es (B.G.-V.); vitoverardo@ugr.es (V.V.) \\ 2 Department of Nutrition and Food Science, University of Granada, C/Santander, 52005 Melilla, Spain; \\ celiarp@ugr.es \\ 3 Institute of Nutrition and Food Technology 'José Mataix', Biomedical Research Center, \\ University of Granada, Avda del Conocimiento sn, 18100 Granada, Spain \\ 4 Department of Analytical Chemistry, University of Granada, Avda Fuentenueva, 18071 Granada, Spain; \\ anagomez@ugr.es \\ * Correspondence: carmenrazola@correo.ugr.es \\ † Presented at the 1st International Electronic Conference on Food Science and Functional Foods, \\ 10-25 November 2020; Available online: https://foods_2020.sciforum.net/.
}

Citation: Razola-Díaz, M.d.C.; Guerra-Hernández, E.J.; RodríguezPérez, C.; Gómez-Caravaca, A.M.; García-Villanova, B.; Verardo, V. Optimization of Ultrasound Assisted Extraction of Phenolic Compounds from Orange By-Product. 2021, 70, 49. https://doi.org/10.3390/ foods_2020-07691

Published: 9 November 2020

Publisher's Note: MDPI stays neutral with regard to jurisdictional claims in published maps and institutional affiliations.

Copyright: $@ 2020$ by the authors. Licensee MDPI, Basel, Switzerland. This article is an open access article distributed under the terms and conditions of the Creative Commons Attribution (CC BY) license (http://creativecommons.org/licenses/by/4.0/).

\begin{abstract}
Orange peel (OP) is the main by-product from orange juice industry. OP is a known source of bioactive compounds and is widely studied for its antioxidant, anti-inflammatory, anti-cancer, anti-rheumatic, anti-diabetic and cardioprotective activities. Thus, this research focuses on the establishments of ultrasound-assisted extraction of phenolic compounds in orange peel using a sonotrode; objective framed in the European SHEALTHY (non-thermal physical technologies to preserve healthiness of fresh and minimally processed fruit and vegetables) project. For this purpose, a Box Behnken design of 27 experiments was carried out with 4 independent factors (ratio ethanol/water, time (min), amplitude (\%) and pulse (\%)). Quantitative analyses of total phenolic compounds (TPC) were performed by Folin-Ciocalteu method and the antioxidant activity was measured by ABTS and DPPH methods. The validity of the experimental design was confirmed by ANOVA and the optimal sonotrode extraction conditions were obtained by response surface methodology. The optimal extracts were characterized by HPLC coupled to mass spectrometer detectors. The highest phenolic content and antioxidant activity was obtained using 45/55 ethanol/water ( $v / v)$, $35 \mathrm{~min}$, amplitude $90 \%(110 \mathrm{~W})$ and pulse $100 \%$. The established method allows the extraction of $30.42 \mathrm{mg}$ of gallic acid equivalents/g dry weight of total phenolic compounds from OP; this value suppose an increment up to $60 \%$ higher than conventional extraction.
\end{abstract}

Keywords: Box-Behnken design; phenolic bioactive compounds; antioxidant activity; orange peel; sonotrode ultrasound assisted extraction; HPLC-MS

\section{Introduction}

Orange is the second most produced fruit in the European Union, mainly in the countries of the Mediterranean basin supposing 6 million tons, almost $10 \%$ of the world production. Increasingly, citrus juices have gained great popularity, representing more than $50 \%$ of the juices available in international trade. Orange juice is the most predominant product processed by the beverage industry and consumed throughout the world due to its high nutritional value and desirable sensory characteristics. The processing of oranges to obtain juices or citrus-based beverages generates large amounts of waste by-products, such as peels, which are a rich source of soluble sugars, phenolic compounds, flavonoids, dietary fibers (cellulose, hemicelluloses, pectin), vitamins and essential oils, so they can 
be used for the production of nutritional dietary supplements at a low cost [1]. The orange peel has been reported to have antioxidant [2], anti-inflammatory [3], anti-cancer [4], antirheumatic [5], anti-diabetic [6] and cardioprotective activities [7], mainly attributed to its content in phenolic compounds. Traditionally, methods based on maceration and thermal extraction with different solvents such as hexane, acetone, methanol, etc. have been used to recover nutritionally valuable compounds from this type of fruit waste [8]. However, the need to increase extraction yields has led to the study of new, more in-depth unconventional methods as ultrasound technology. These methods can reduce extraction time and have lower temperature and solvent consumption, as well as achieve higher efficiency and lower energy consumption compared to conventional methods.

\section{Materials and Methods}

\subsection{Chemicals and Samples}

Gallic acid, DPPH and ABTS were purchased from Sigma-Aldrich (St. Louis, MO, USA). $\mathrm{Na}_{2} \mathrm{CO}_{3}$ purchased from BDH AnalaR (Poole, UK). Double-deionised water was from Millipore (Bedford, MA, USA). HPLC-grade water, Folin-Ciocalteu reagent and other reagents were purchased from Merck KGaA (Darmstadt, Germany).

Orange by-product was obtained from the company Ekolo (Navarra, Spain) after cutting the orange in two pieces and pressing them mechanically to obtain juice. The resulting by-product is composed by the albedo, flavedo and rests of pulp of the orange with a humidity of $70 \pm 1.5 \%$. The samples were dried and grinded, and frozen at $-18{ }^{\circ} \mathrm{C}$ until the analyses.

\subsection{Experimental Design}

The conditions for obtaining the highest phenolic compounds and antioxidant activity from the orange peel has been optimized by using Box-Behnken design and response surface methodology (RSM). The designs are composed by 27 experiment structured in three blocks with three levels $(-1,0,1)$. Each experiment has been carried out in duplicate. The independent variables are ratio ethanol/water $(0 / 100,50 / 50,100 / 0 v / v)$, time $(5,25,45$ min), amplitude $(20,60,100 \%)$, and pulse $(10,50,100 \%)$. The dependent variables have been adjusted to a second order polynomial model equation (Equation (1)), where $\Upsilon$ represents the response variable, the total phenolic compounds (TPC), or the antioxidant assays ABTS or DPPH, $X_{i}$ and $X_{j}$ are the independent factors that affect the response and $\beta_{0}$, $\beta_{\mathrm{i}}, \beta_{\mathrm{ii}}$ and $\beta_{\mathrm{ij}}$ are the regression coefficients of the model (interception, linear, quadratic and interaction term). The Statistica 7.0 package (StatSoft, Tulsa, OK, USA) has been used for the mathematical operations and simulations.

Equation (1) Second order polynomial equation.

$$
\Upsilon=\beta_{0}+\sum_{\mathrm{i}=0}^{4} \beta_{\mathrm{i}} \mathrm{X}_{\mathrm{i}}+\sum_{\mathrm{i}=0}^{4} \beta_{\mathrm{ii}} \mathrm{X}_{\mathrm{ii}}^{2}+\sum_{\mathrm{i}=0}^{4} \sum_{\mathrm{j}=0}^{4} \beta_{\mathrm{ii}} \mathrm{X}_{\mathrm{i}} \mathrm{X}_{\mathrm{j}}
$$

The ANOVA assay has been performed in order to evaluate the adjustment of the models having into account the regression coefficients, the $p$-values of the regression, the lack of adjustment. The optimum conditions were established using RSM.

\subsection{Extraction of Phenolic Compounds from Orange Peel by Sonotrode Ultrasonic Extraction}

It was weight $0.5 \mathrm{~g}$ of sample and a ratio of ethanol/water $(v / v)$ up to $100 \mathrm{~mL}$ was added in a $250 \mathrm{~mL}$ beaker. It was introduced the sonotrode (UP400St ultrasonic processor, Hielscher, Germany) with previous configuration of the pulse and amplitude, and the sample was sonicated during a determined time. Once the time was finished, the flasks were centrifugated in Falcon tubes and the supernatant was collected and allowed at -18 ${ }^{\circ} \mathrm{C}$ till analyses. 


\subsection{Conventional Extraction of Phenolic Compounds}

The conventional extraction has been carried out with the optimal ratio ethanol/water and time. It was weight $0.5 \mathrm{~g}$ of sample and a ratio of ethanol/water $(v / v)$ up to $100 \mathrm{~mL}$ was added in a $250 \mathrm{~mL}$ beaker. It was agitated by a magnetic stirrer during a determined time and after that the flasks were centrifugated in Falcon tubes and the supernatant was collected and allowed at $-18^{\circ} \mathrm{C}$ till analyses.

\subsection{Determination of Phenolic Compounds}

The total phenolic compounds has been determined by the Folin-Ciocalteu spectrophotometric method [9]. It was taken $100 \mu \mathrm{L}$ of the extract in a volumetric flask of $10 \mathrm{~mL}$ and it was added of $500 \mu \mathrm{L}$ of the Folin-Ciocalteu reagents and $6 \mathrm{~mL}$ of bi-distilled water. The flask was agitated for a minute. After that, $2 \mathrm{~mL}$ of $15 \%(w / v) \mathrm{Na}_{2} \mathrm{CO}_{3}$ was added and the volumetric flasks were flushed and maintained hidden from the light. The measured were carried out after $2 \mathrm{~h}$ at $750 \mathrm{~nm}$ and $25^{\circ} \mathrm{C}$ with a UV-visible spectrophotometer (Spectrophotometer 300 Array, UV-Vis, single beam, Shimadzu, Duisburg, Germany). The results were compared to a standard curve of gallic acid equivalents $(1,5,10,25,50,100,250$ ppm) to calculate the total phenolics compounds (TPC). Results are expressed as mg gallic acid equivalents (GAE)/g dry weight (d.w.). The optimal extracts have been characterized by HPLC coupled to mass spectrometer detectors.

\subsection{Antioxidant Assays}

Also, the antioxidant capacity has been evaluated in the 27 experiments twice by two different methods. The first one is the developed by Re et al., (1999) [10] in which the monocation $\mathrm{ABTS}^{\bullet+}$ is generated by oxidation of the ABTS with potassium persulfate in the dark at room temperature for a $12-24 \mathrm{~h}$. For each extract, it was added $1 \mathrm{~mL}$ of this ABTS solution to $0.01 \mathrm{~mL}$ of extract and it was measured the detriment of absorbance during $10 \mathrm{~min}$ at $734 \mathrm{~nm}$. The results were compared with a standard curve of Trolox equivalents $(1,10,20,50,80,100,150,200$ ppm). On the other hand, the DPPH radical scavenging activity was assayed with a method proposed by several authors [11,12]. 100 $\mu \mathrm{L}$ of each extract was added of $2.9 \mathrm{~mL}$ of $\mathrm{DPPH}$, and after rapid stirring, the bleaching power of the extract was observed in a time interval from 0 to $30 \mathrm{~min}$ at $517 \mathrm{~nm}$. The results were compared to a curve of Trolox equivalents in methanol/water 4/1 (v/v) (1, 10, 20, 50, 80, 100, 150, $200 \mathrm{ppm})$. Results for both assays are expressed as mg Trolox equivalents (TE)/g dry weight (d.w.).

\section{Results and Discussion}

\subsection{Determination of Phenolic Compounds and Antioxidant Capacity in Orange Peel}

The Box-Behnken model coupled to response surface methodology have been used to optimize the extraction of phenolic compounds with the higher antioxidant capacity. For the total phenolic compounds, the observed values ranged between 8.66 and $29.75 \mathrm{mg}$ GAE/g d.w. The lower one corresponds to the condition $100 \%$ ethanol/water $(v / v), 25 \mathrm{~min}$, $20 \%$ amplitude and 50\% cadence, and the highest one to $50 \%$ ethanol/water $(v / v), 25 \mathrm{~min}$, $100 \%$ amplitude and $100 \%$ cadence.

For the antioxidant assays, it ranged from 9.54 to $26.45 \mathrm{mg}$ TE/g d.w. with the DPPH technique, and with the ABTS technique the range obtained was $11.46-40.52 \mathrm{mg}$ TE/g d.w. The minimum value with both methods was obtained using only ethanol $100 \%(v / v)$, meanwhile the highest ones by using a mix of $50 \%$ ethanol and $50 \%$ water $(v / v)$.

\subsection{Fitting the Model}

The experimental data was analyzed adjusting it to a second order polynomial equation, a regression model that, using the least squares methods, provides the lowest residual value. The non-significant terms with a significance level of $p<0.05$ were discarded, and the model was recalculated only with significant terms. For the three responses it was 
revealed a moderately high correlation between the factors and the response variable $\left(R^{2}\right.$ $=0.9564,0.9286$ and 0.8694 for TPC, ABTS and DPPH, respectively). The validity of the model was tested with ANOVA, which showed that, the model can be statistically accepted because the regression $p$ values are lower than 0.05 and the lacks of fit for the three variables are non-significant $(p>0.05)$. Taking into account the regression coefficients, for the 3 response variables the individual linear factors ratio ethanol/water $\left(\beta_{1}\right)$, time $\left(\beta_{2}\right)$ and pulse $\left(\beta_{4}\right)$ showed significant effects. For the TPC and DPPH, the crossed interaction $\beta_{12}$ was significant, and the crossed interactions $\beta_{23}$ and $\beta_{34}$ were significant for the TPC and ABTS. Moreover, the quadratic term $\beta_{11}$ was significant for the three response variables, meanwhile $\beta_{22}$ was significant for ABTS and DPPH, and $\beta_{44}$ only for TPC. The amplitude $\left(\beta_{3}\right)$ and the rest of interactions non mentioned did not have significant effect in the meanings chosen.

\subsection{Confirmation of Optimal Sonotrode Ultrasound Parameters}

The used software does not provide the critical values, so it has been established a compromise between the independent factors and the three dependent variables in order to maximize the responses. The optimal conditions have been selected by RSM through three-dimensional plots, looking for same conditions for the three variables (Table 1).

Table 1. Optimal conditions selected and the model predicted values with the obtained values.

\begin{tabular}{cccc}
\hline Parameter & \multicolumn{3}{c}{ Optimal Conditions } \\
\hline Ethanol/water $(\%(v / v))$ & & 45 & \\
Time (min) & & 35 & \\
Amplitude (\%) & & 90 & \\
Pulse (\%) & TPC & DPPH & ABTS \\
\hline & $29.36 \pm 3.5$ & $24.44 \pm 3.6$ & $32.02 \pm 7.0$ \\
\hline Predicted value (mg/g d.w.) & $30.42 \pm 1.5$ & $26.37 \pm 1.6$ & $35.62 \pm 2.1$ \\
\hline Obtained value (mg/g d.w.)
\end{tabular}

Briefly, optimal sonotrode extraction conditions were $45 \%$ ethanol/water $(v / v), 35$ min, amplitude $90 \%(110 \mathrm{~W})$, and pulse $100 \%$. Verifying the accuracy of the mathematical model, the obtained values did not report significant differences with the predicted values in the case of the total phenolic compounds, and the antioxidant assays DPPH and ABTS. According to the results, ethanol was not an efficient solvent when used pure, showing better results when it was combined with water in a proportion equal or minor to $50 \%$. This occurs due to the increased solvation provided by the presence of water. Additionally, the time has been chosen as lower as possible in order to develop a quick procedure. The highest values of amplitude and pulse has been demonstrated to be necessary in order to reach higher powers and finally obtain better performances.

With the conventional extraction it was obtained $17.76 \mathrm{mg}$ GAE/g d.w. According to the results obtained, the proposed optimal methodology using sonotrode let recover $60 \%$ more total phenolic compounds. Other authors have been optimized the conventional extraction of the phenolic compounds agreeing that the higher the temperature and time, the greater is the total phenol recovery from the orange peel. Hernández-Carranza et al. (2015) needed $60^{\circ} \mathrm{C}$ during $12 \mathrm{~h}$ using water as solvent for obtaining $6.89 \mathrm{mg} \mathrm{GAE} / \mathrm{g} \mathrm{d} . \mathrm{w}$. [13]. Using methanol as solvent Victor et al., (2020) obtained $28 \mathrm{mg}$ GAE/g d.w. needing 3 $\mathrm{h}$ at $55^{\circ} \mathrm{C}$ [14], and Lagha-Benamrouche et al., (2013) obtained $25.60 \mathrm{mg}$ GAE/g d.w. needing $22 \mathrm{~h}$ at $20^{\circ} \mathrm{C}$ [15]. Also, other author has used ultrasound technology to optimize the extraction of phenolic compounds from the orange peel. Barrales et al., (2018) [16] used ultrasonic bath with ethanol $50 \%(v / v)$ during 15 min obtaining $5.5 \mathrm{mg} \mathrm{GAE} / \mathrm{g}$ d.w. LachosPérez et al., (2018) [17] used sonotrode with ethanol $80 \%(v / v)$ during $2 \mathrm{~h}$ at $800 \mathrm{~W}$ obtaining 5.83 mg GAE/g d.w. Dahmoune et al., (2014) [18] and Nayak et al., (2015) [19] also 
used sonotrode but with acetone $75.79 \%(v / v), 8.33 \mathrm{~min}$ and amplitude $65.94 \%$, and obtained 13.57 and $10.35 \mathrm{mg}$ GAE/g d.w., respectively. In general, the proposed sonotrode optimized conditions let obtain higher recovery of total phenolic compounds than all the references that use conventional extraction or ultrasound technology, with lower times and temperature.

The optimal extracts were characterized by HPLC, and the majority identified compound is hesperidin and account at least $35 \%$ of the present flavonoids. The other majority flavonoid identify is narirutin. Ferulic and caffeic acids derivates are the majority phenolic acids identify. All the identified compounds corroborate the results obtained by Karoui et al., (2013) [20], Chen et al., (2012) [21], Toledo-Guillén et al., (2010) [22], and MonteroCalderón et al., (2019) [23].

In the bibliography the major part of the works uses the DPPH technique instead of the others. The optimal value obtained for DPPH is higher than those obtained by Hernández-Carranza et al., (2016) (8.94 mg TE/g d.w.) [13] and Barrales et al., (2018) (4.30 mg TE/g d.w.) [16]. However, Lachos-Pérez et al., (2018) [17] obtained an optimal result slightly higher (26.78 mg TE/g d.w.) but with a time of $2 \mathrm{~h}$, much higher that the optimized conditions.

Author Contributions: All authors have read and agree to the published version of the manuscript. Conceptualization, V.V. and E.J.G.-H.; investigation, M.d.C.R.-D.; data curation, M.d.C.R.-D., A.M.G.-C. and C.R.-P.; writing-original draft preparation, M.d.C.R.-D.; writing-review and editing, V.V., E.J.G.-H., B.G.-V.; supervision, V.V. and E.J.G.-H.; funding acquisition, V.V. All authors have read and agreed to the published version of the manuscript.

Funding: This study is part of the SHEALTHY project that has received funding from European Union's Horizon 2020 research and innovation program under grant agreement No 817936.

Acknowledgments: Vito Verardo thanks the Spanish Ministry of Economy and Competitiveness (MINECO) for "Ramon y Cajal" contract (RYC-2015-18795).

Conflicts of Interest: The authors declare no conflict of interest.

\section{References}

1. El Kantar, S.; Boussetta, N.; Rajha, H.N.; Maroun, R.G.; Louka, N.; Vorobiev, E. High voltage electrical discharges combined with enzymatic hydrolysis for extraction of polyphenols and fermentable sugars from orange peels. Food Res. Int. 2018, 107, 755-762.

2. Wang, S.; He, N.; Xing, H.; Sun, Y.; Ding, J.; Liu, L. Function of hesperidin alleviating inflammation and oxidative stress responses in COPD mice might be related to SIRT1/PGC-1 $\alpha /$ NF- $\kappa B$ signaling axis. J. Recept. Signal Transduct. 2020, 40, 388-394.

3. Liu, L.; Shan, S.; Zhang, K.; Ning, Z.-Q.; Lu, X.-P.; Cheng, Y.-Y. Naringenin and hesperetin, two flavonoids derived from Citrus aurantium up-regulate transcription of adiponectin. Phyther. Res. 2008, 22, 1400-1403.

4. Choi, E.J.; Kim, G.H. Anti-/pro-apoptotic effects of hesperetin against 7,12-dimetylbenz(a) anthracene-induced alteration in animals. Oncol. Rep. 2011, 25, 545-550.

5. Guardia, T.; Rotelli, A.E.; Juarez, A.O.; Pelzer, L.E. Anti-inflammatory properties of plant flavonoids. Effects of rutin, quercetin and hesperidin on adjuvant arthritis in rat. Farmaco 2001, 56, 683-687.

6. Mahmoud, A.M.; Ashour, M.B.; Abdel-Moneim, A.; Ahmed, O.M. Hesperidin and naringin attenuate hyperglycemia-mediated oxidative stress and proinflammatory cytokine production in high fat fed/streptozotocin-induced type 2 diabetic rats. J. Diabetes Complicat. 2012, 26, 483-490.

7. Elavarasan, J.; Velusamy, P.; Ganesan, T.; Ramakrishnan, S.K.; Rajasekaran, D.; Periandavan, K. Hesperidin-mediated expression of Nrf2 and upregulation of antioxidant status in senescent rat heart. J. Pharm. Pharmacol. 2012, 64, 1472-1482.

8. Wang, L.; Weller, C.L. Recent advances in extraction of nutraceuticals from plants. Trends Food Sci. Technol. 2006, 17, 300-312.

9. Singleton, V.L.; Orthofer, R.; Lamuela-Raventós, R.M. Analysis of Total Phenols and other oxidation substrates and antioxidants by means of Folin-Ciocalteu Reagent. Methods Enzymol. 1974, 299, 152-178.

10. Re, R.; Pellegrini, N.; Proteggente, A.; Pannala, A.; Yang, M.; Rice-Evans, C. Antioxidant activity applying an improved ABTS radical cation decolorization assay. Free Radic. Biol. Med. 1999, 26, 1231-1237.

11. Brand-Williams, W.; Cuvelier, M.E.; Berset, C. Use of a free redical method to evaluate antioxidant activity. LWT Food Sci. Technol. 1995, 28, 25-30.

12. Parejo, I.; Codina, C.; Petrakis, C.; Kefalas, P. Evaluation of scavenging activity assessed by Co(II)/EDTA-induced luminol chemiluminescence and DPPH (2,2-diphenyl-1-picrylhydrazyl) free radical assay. J. Pharmacol. Toxicol. Methods 2000, 44, 507-512. 
13. Hernández-Carranza, P.; Ávila-Sosa, R.; Guerrero-Beltrán, J.A.; Navarro-Cruz, A.R.; Corona-Jiménez, E.; Ochoa-Velasco, C.E. Optimization of Antioxidant Compounds Extraction from Fruit By-Products: Apple Pomace, Orange and Banana Peel. J. Food Process. Preserv. 2016, 40, 103-115.

14. Victor, M.M.; David, J.M.; Cortez, M.V.M.; Leite, J.L.; da Silva, G.S.B. A High-Yield Process for Extraction of Hesperidin from Orange (Citrus sinensis L. osbeck) Peels Waste, and Its Transformation to Diosmetin, A Valuable and Bioactive Flavonoid. Waste Biomass Valoriz. 2020, doi:10.1007/s12649-020-00982-x.

15. Lagha-Benamrouche, S.; Madani, K. Phenolic contents and antioxidant activity of orange varieties (Citrus sinensis L. and Citrus aurantium L.) cultivated in Algeria: Peels and leaves. Ind. Crops Prod. 2013, 50, 723-730.

16. Barrales, F.M.; Silveira, P.; Barbosa, P. de P.M.; Ruviaro, A.R.; Paulino, B.N.; Pastore, G.M.; Macedo, G.A.; Martinez, J. Recovery of phenolic compounds from citrus by-products using pressurized liquids-An application to orange peel. Food Bioprod. Process. 2018, 112, 9-21.

17. Lachos-Perez, D.; Baseggio, A.M.; Mayanga-Torres, P.C.; Maróstica, M.R.; Rostagno, M.A.; Martínez, J.; Forster-Carneiro, T. Subcritical water extraction of flavanones from defatted orange peel. J. Supercrit. Fluids 2018, 138, 7-16.

18. Dahmoune, F.; Moussi, K.; Remini, H.; Belbahi, A.; Aoun, O.; Spigno, G.; Madani, K. Optimization of ultrasound-assisted extraction of phenolic compounds from citrus sinensis L. peels using response surface methodology. Chem. Eng. Trans. 2014, 37, 889-894.

19. Nayak, B.; Dahmoune, F.; Moussi, K.; Remini, H.; Dairi, S.; Aoun, O.; Khodir, M. Comparison of microwave, ultrasound and accelerated-assisted solvent extraction for recovery of polyphenols from Citrus sinensis peels. Food Chem. 2015, 187, 507-516.

20. Jabri Karoui, I.; Marzouk, B. Characterization of bioactive compounds in Tunisian bitter orange (Citrus aurantium L.) peel and juice and determination of their antioxidant activities. Biomed Res. Int. 2013, 2013.

21. Chen, Z.T.; Chu, H.L.; Chyau, C.C.; Chu, C.C.; Duh, P. Der Protective effects of sweet orange (Citrus sinensis) peel and their bioactive compounds on oxidative stress. Food Chem. 2012, 135, 2119-2127.

22. Toledo-Guillén, A.R.; Higuera-Ciapara, I.; García-Navarrete, G.; de la Fuente, J.C. Extraction of Bioactive Flavonoid Compounds from Orange (Citrus sinensis) Peel Using Supercritical CO2. J. Biotechnol. 2010, 150, 313-314.

23. Montero-Calderon, A.; Cortes, C.; Zulueta, A.; Frigola, A.; Esteve, M.J. Green solvents and Ultrasound-Assisted Extraction of bioactive orange (Citrus sinensis) peel compounds. Sci. Rep. 2019, 9, 1-8. 